\title{
A Method For Detecting Displacement Front Based On Ultrasonic Velocity
}

\author{
Chenquan Hua ${ }^{1, a}$,Xinyue Che ${ }^{1, b}$, Jibo Wang $^{1, c}$ and Hongwei Huang ${ }^{1, d}$ \\ ${ }^{1}$ College of Information \& Control Engineering, China University of Petroleum, Qingdao, China. \\ ahcq7105@163.com, b chexinyue1993@qq.com, c 875427223@qq.com, d1134203663 @qq.com
}

Keywords: displacement front, ultrasonic velocity, first break time, catastrophe point Abstract: A noninvasive method based on ultrasonic velocity was proposed to detect the displacement front in core displacement experiments. $\mathrm{CO} 2$ drive experiments were carried out to prove the possibility of detecting the displacement front. The characteristic that ultrasounds have different propagation velocities in different media was used to detect the displacement front in core displacement experiments. The average velocity can be calculated by measuring the first break time of the ultrasonic wave. Arranging all average velocities of different times at a certain position of the core model in chronological order and then finding the catastrophe point, it is the moment of the catastrophe point that the displacement front arrived.

\section{Introduction}

As a modern detection technology, ultrasonic detection has been applied more and more widely. It is known that ultrasonic detection technology is noninvasive, nondestructive and harmless when compared with other monitoring techniques [1]. Plenty of detections in various fields can be carried out using the characteristic that ultrasounds have different propagation velocities and attenuation in different media [2] [3]. This paper utilized the characteristic of ultrasonic velocity [4] [5].

Chou Y S et al investigated and proposed a novel method for monitoring the state of charge of a vanadium redox flow battery(VRFB) by using ultrasonic velocity [6]. Voleisis A et al researched an approach to monitoring the clotting process during whole blood coagulation by using ultrasonic velocity [7]. Zhang $\mathrm{Y}$ et al concluded that using ultrasonic velocity to quantify deformation-induced damage evolution in PE plates can be a nondestructive means [8].

In this paper, a noninvasive method based on ultrasonic velocity was proposed to detect the displacement front in core displacement experiments.

\section{Theory of detection}

A Fig. 1 shows the characteristic that ultrasounds have different propagation velocities in different media. The $t_{1}$ and $t_{2}$ in Fig. 4 represent the first break time of two waves. In the case that the height of the core model is known, the average velocity of ultrasound at a certain position and a certain time can be calculated by measuring the first break time. The first break time $\mathrm{T}_{0}$ is shown as equation 1 :

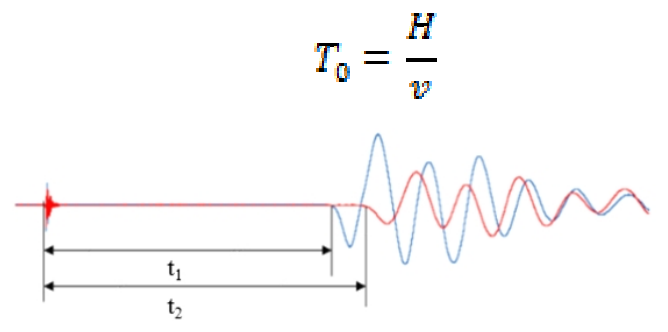

Fig. 1. Schematic of ultrasonic time difference.

where $\mathrm{H}$ and $\mathrm{v}$ are the height of the core model and the ultrasonic propagation velocity in a certain medium, respectively. 
In the case that the height is known $(6 \mathrm{~cm}), \mathrm{T}_{0}$ depends on the velocity of ultrasound in fluids. On account that ultrasounds have different propagation velocities in different media, at some point, the average velocity at a certain position would change suddenly if the displacement front arrived at this position. Therefore, by measuring the first break times we could calculate the average velocities at different times at a certain position. Arranging all average velocities of a certain position in chronological order and then finding the catastrophe point, it is the moment of the catastrophe point that the displacement front arrived.

\section{Experimental setup}

Fig. 2 shows the schematic of the experimental setup in our experiment and study. The apparatus consists mainly of four units: the ultrasonic transmitting unit, the core model, the ultrasonic receiver unit and the central control unit. The ultrasonic transmitting unit consists of the high voltage signal generator and the multiway switch, this unit generates appropriate ultrasound to enter into the core model. The core model is made from sandstones $(30 \mathrm{~cm}$ in length, $10 \mathrm{~cm}$ in width and $6 \mathrm{~cm}$ in height, $30 \%$ porosity). Fig. 2 also shows that there are 12 pairs of probes in total distributed equably on the surface of the core. The core model in figure 1 shows that the transmitting probes are placed on the upper surface of the core and the receiver probes are placed on the lower surface. Coupling agents are smeared between all probes and the surface of the core. The center frequency of the probes is $300 \mathrm{kHz}$. The ultrasonic receiver unit consists of the multiway switch and the data acquisition unit, this unit receives the waveforms penetrated from the core and saves all signals of the whole experimental procedure. The central control unit controls and coordinates other units as well as realizes the automation of experimental procedure.

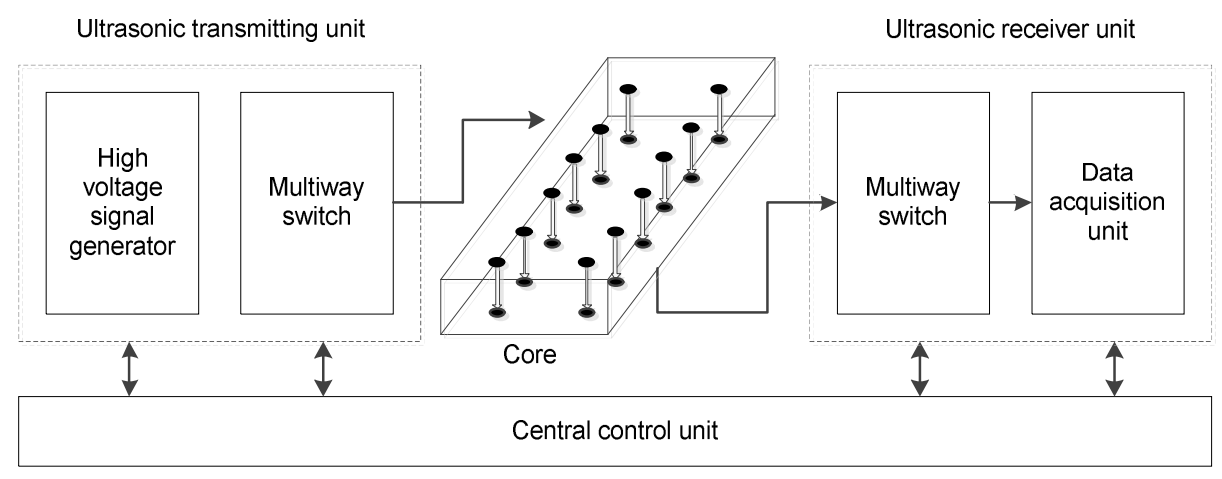

Fig. 2. Schematic of experimental setup.

Step 1. Core model vacuuming. The core sample was first dried at room temperature for several days and then vacuumed for six to eight hours. After the core sample was vacuumed, increased the confining pressure to 13MPa slowly [9].

Step 2. Water saturation. The purpose of this step is to get the porosity and the volume of pore from the volume of the saturated water.

Step 3. Oil saturation. Mimetic oil (black oil) was injected into the core sample until there was nearly no water flowed out from the core sample $\left(60^{\circ} \mathrm{C}\right.$ in temperature and $11 \mathrm{MPa}$ in pressure). The purpose of this step is to make the core sample oil-saturated and build bound water.

Step 4. $\mathrm{CO}_{2}$ drive. At last, supercritical $\mathrm{CO}_{2}\left(\mathrm{CO}_{2}\right.$ has a critical temperature and pressure of $31^{\circ} \mathrm{C}$ and 7.4MPa [10] [11].) was injected into the core sample to drive the oil out.

\section{Results and discussion}

Fig. 3 shows eight ultrasonic signals of a probe at different times in the process of $\mathrm{CO}_{2}$ drive. It can be seen from Fig. 5 that the first waves moved right with the increase of time. That is to say, the first break times $T_{0}$ increased as time went on. What calls for special attention is that the ultrasonic signals will move right suddenly if the displacement front arrive at the position of the probe. 
Fig. 4 shows the trend chart of average velocities of a probe in $\mathrm{CO}_{2}$ drive. As the ultrasonic propagation velocity in $\mathrm{CO}_{2}$ is far less than that in oil, the first break times $T_{0}$ will increase on the basis of equation 1, this is consistent with the result of figure 5. Therefore, the average velocity will decrease as figure 6 shows. What's more, as shown in figure 6 , the moment of the catastrophe point is 9:25. Hence, the moment that the displacement front of $\mathrm{CO}_{2}$ drive arrived is 9:25.

However, it should be noted that if there is no catastrophe point in the trend chart of average velocities, this means that the displacement front did not arrive at this position.

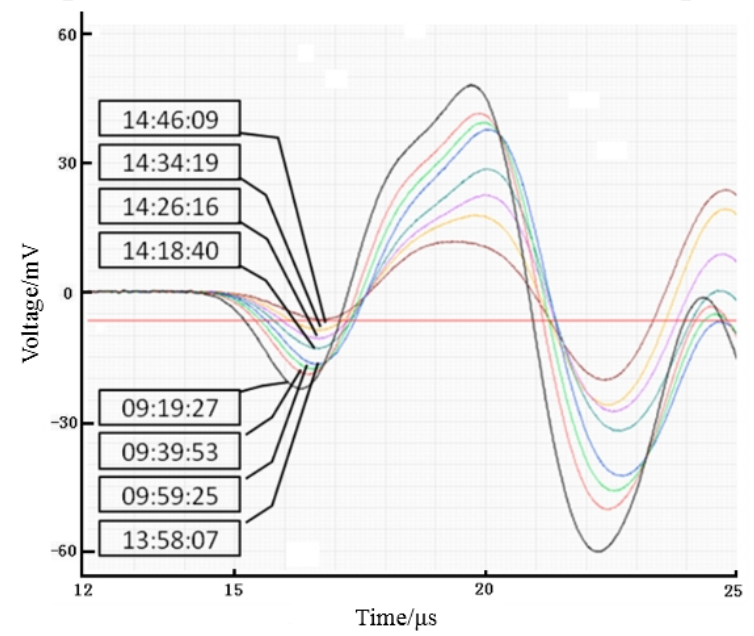

Fig. 3. Ultrasonic signals of a probe at different times in $\mathrm{CO}_{2}$ drive.

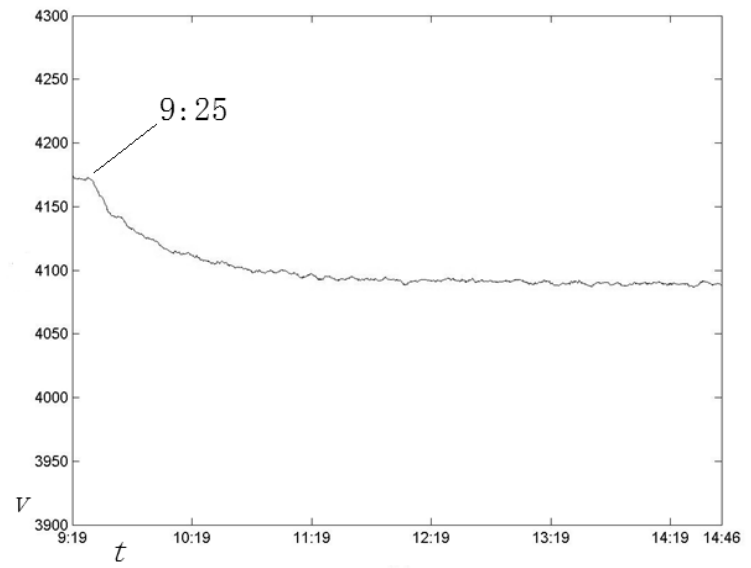

Fig. 4. Trend chart of average velocities of a probe in $\mathrm{CO}_{2}$ drive.

\section{Conclusions}

Ultrasonic methods are widely agreed to be one of the most promising tools for detecting and monitoring the process of experiments. This paper provides a possibility to detect the displacement front in core displacement experiments. The displacement manners are not restricted to $\mathrm{CO}_{2}$ drive, it is also appropriate for water flooding, $\mathrm{CO}_{2}$ sequestration, polymer flooding and so on.

\section{Acknowledgements}

This work was financially supported by the ShanDong Natural Science Foundation (ZR2016EEM46) and Industry-University Cooperation Project of Ministry of Education (201602008017). 


\section{References}

[1] Budelli, Eliana, et al. "Evaluation of ultrasonic techniques for on line coagulation monitoring in cheesemaking." Journal of Food Engineering 209(2017):83-88.

[2] Diallo, M. S., M. Prasad, and E. Appel. "Comparison between experimental results and theoretical predictions for P-wave velocity and attenuation at ultrasonic frequency." Wave Motion 37.1(2003):1-16.

[3] Mashinskii, E. I. "Strain amplitude-dependent attenuation of and waves in dry and water-saturated sandstone under confining pressure." Russian Geology \& Geophysics 50.8(2009):734-738.

[4] Shi, Ji Quan, Z. Xue, and S. Durucan. "Seismic monitoring and modelling of supercritical CO 2, injection into a water-saturated sandstone: Interpretation of P-wave velocity data." International Journal of Greenhouse Gas Control 1.4(2007):473-480.

[5] Liu, J. W., et al. "Velocity-saturation relation in partially saturated rocks: Modelling the effect of injection rate changes." Eage Conference and Exhibition 2016.

[6] Chou, Yi Sin, et al. "A novel ultrasonic velocity sensing approach to monitoring state of charge of vanadium redox flow battery." Applied Energy 182(2016):253-259.

[7] Voleisis, A, et al. "Ultrasonic method for monitoring the clotting process during whole blood coagulation. " Ultrasonics 78(2017):146-151.

[8] Zhang, Yi, et al. "Characterization of ductile damage in polyethylene plate using ultrasonic testing." Polymer Testing 62(2017):51-60.

[9] Lei, Xinglin, and Z. Xue. "Ultrasonic velocity and attenuation during $\mathrm{CO} 2$, injection into water-saturated porous sandstone: Measurements using difference seismic tomography." Physics of the Earth \& Planetary Interiors 176.3(2009):224-234.

[10] Xue, Z, and T. Ohsumi. "Seismic wave monitoring of CO2 migration in water-saturated porous sandstone." Exploration Geophysics 35.1(2004):25-32.

[11] Lebedev, Maxim, et al. "An experimental study of acoustic responses on the injection of supercritical into sandstones from the Otway Basin." Geophysics 78.4(2013):293-306. 